\title{
ONLINE FATIGUE CRACK GROWTH MONITORING WITH CLIP GAUGE AND DIRECT CURRENT POTENTIAL DROP
}

\author{
S. De Tender, N. Micone and W. De Waele \\ Ghent University, Laboratory Soete, Belgium
}

\begin{abstract}
Fatigue is a well-known failure phenomenon which has been and still is extensively studied. Often structures are designed according to the safe-life principle so no crack initiation occurs. Nowadays there is a high emphasis on cost-efficiency, and one might rather opt for a fail-safe design. Therefore a certain amount of crack growth can be allowed in structures, but then a good knowledge of stresses and related crack growth rates is needed. To this end, extensive studies are done to obtain a material's Paris law curve. Within the framework of research for offshore wind turbine constructions, tests were done to determine the crack growth rate of a high strength low alloy (HSLA) steel. A dedicated LabVIEW program was developed to be able to determine an entire Paris law curve with a single specimen, by controlling the stress intensity factor range $(\Delta \mathrm{K})$. The program is controlled by the readings of a clip gauge, which make it possible to plan the amount of crack growth per $\Delta \mathrm{K}$ block and thus plan an entire test in advance. The potential drop technique was also applied in order to obtain the Paris law curve. Clip gauge results were compared with direct current potential drop monitoring. This comparison was done by means of an a/W-N diagram and the resulting Paris law curves. The results show a very good correlation between both methods and with the visual confirmation.
\end{abstract}

Keywords: $\Delta \mathrm{K}, \mathrm{da} / \mathrm{dN}$, Paris law curve, a/W-N curve, clip gauge, DCPD, K-decreasing, K-increasing

\section{NOMENCLATURE}

$\begin{array}{llllll}d a / d N & \text { crack growth rate } & m m / c y c l e & B & \text { Specimen Thickness } & m m \\ K & \text { stress intensity factor } & M P a * \sqrt{m} & W & \text { Specimen Width } & \mathrm{mm} \\ P & \text { Force } & N & E & \text { Young's Modulus } & \mathrm{GPa} \\ \Delta a & \text { crack length } & m m & v_{0} & \text { Crack mouth opening } & \mathrm{mm} \\ f & \text { frequency } & \mathrm{Hz} & & \text { Displacement } & \\ R & \text { stress ratio } & - & V & \text { Voltage } & \mathrm{V} \\ \sigma_{y} & \text { yield strength } & \mathrm{MPa} & Y_{0} & \text { Measurement pin } & \mathrm{mm} \\ \sigma_{\text {uts }} & \text { Ultimate tensile strength } & \mathrm{MPa} & & \text { distance } & \end{array}$

\section{INTRODUCTION}

Fatigue can be investigated in many different ways. In practice, the S-N curve approach is the most popular to represent material characteristics. In this kind of diagram, a certain lifetime is specified for every different constant amplitude stress level. Some applications however, might require that a certain amount of crack growth is allowed, to make them cost efficient. In this case a Paris law curve is often used to define the crack growth rate as a function of stress intensity factor range [1,2]. As shown in figure 1 (right), crack growth rate is described as the increment in crack growth per increment in cycles (da/dN). The stress intensity factor range $(\Delta K)$ is proportional with the force range $(\Delta P)$, depends on geometrical parameters of the used specimen and on the crack length. The curve of a typical steel consists of three parts: the initiation phase (I), the stable propagation phase (II) and the critical propagation phase (III). The initiation phase has as a lower limit the threshold stress intensity factor range (defined below). The stable propagation phase starts and ends when the crack growth rate becomes linear with respect to stress intensity factor range. The critical propagation phase starts when there is crack growth rate acceleration [1].

Another way of representing crack growth in a material is by plotting the relative crack depth $(a / W)$ versus the number of cycles (see fig. 1 (left) [2]). This is an easy way of comparing different kinds of instrumentation and verifying their output with a visual confirmation. 



Figure 1: a/W-N curve (left) with K-decreasing (black) and K-increasing (blue), Paris law curve (right)

ASTM E647 ([3]), which is the standard test method for measurement of fatigue crack growth rates, describes how the Paris law curve should be determined. The standard recommends a minimum precrack length based on geometrical parameters, and a maximum precrack growth rate (da/dN $<10^{-5} \mathrm{~mm} /$ cycle). The threshold region is determined with a $\mathrm{K}$-decreasing method. $\Delta \mathrm{K}$ values are decreased until a crack growth rate lower than $10^{-7} \mathrm{~mm} /$ cycle is reached, this is region I in the right part of figure 1 and the black part in the left figure. When going from precracking to K-decreasing it is important to stay under the maximum stress intensity factor of the precracking stage. Besides there should be sufficient crack growth $(\Delta a)$ per block such that there are as limited transient effects between blocks as possible. For determining the stable propagation phase, a Kincreasing procedure is used. $\Delta \mathrm{K}$ blocks are increased up to the plastic region, which is shown as region II in the right part of figure 1 and as the blue part of the left figure. Again a significant $\Delta$ a should be used per $\Delta \mathrm{K}$ block to keep transient effects as low as possible and to have da/dN values which are as stable as possible.

The goal of the tests are on the one side to obtain a Paris law curve for a certain material based on a clip gauge controlled test. For every different $\Delta \mathrm{K}$ block in both the K-decreasing and increasing modules 4 or 5 average da/dN measurements are taken over a certain crack extent. This crack growth is measured online by means of a clip gauge. These points are then set out in the Paris law diagram. On the other side clip gauge output is compared with direct current potential drop (DCPD) measurements and a visual confirmation. DCPD is a method that is more and more used in fatigue applications, because of its flexibility for geometries and environments [4,5]. It has therefore a wide range of possible applications. The results of this technique are compared with the clip gauge measurement by means of both an a/W-N curve and their responding Paris law curves.

\section{EXPERIMENTAL PROCEDURE}

\subsection{Material}

The steel that is used is similar to an offshore grade NV F460, which is a typical HSLA steel. Table 1 denotes the microstructural properties of the material and table 2 gives the mechanical properties [6].

Table 1

\begin{tabular}{|c|c|c|c|c|c|c|c|c|c|c|}
\hline Material & & $\mathbf{C}$ & $\mathbf{M n}$ & $\mathbf{S i}$ & $\mathbf{P}$ & $\mathbf{S}$ & $\mathbf{C u}$ & $\mathbf{N i}$ & $\mathbf{C r}$ & $\mathbf{M o}$ \\
\hline NV F460 & {$[\%]$} & 0.08 & 1.24 & 0.24 & 0.01 & 0.001 & 0.05 & 0.21 & 0.05 & 0.005 \\
\hline
\end{tabular}

Table 2

\begin{tabular}{|c|c|c|}
\hline Material & $\sigma_{\mathrm{y}}[\mathrm{MPa}]$ & $\boldsymbol{\sigma}_{\text {uts }}[\mathrm{MPa}]$ \\
\hline NV F460 & 520 & 603 \\
\hline
\end{tabular}




\subsection{Geometry}

The stress intensity factor depends on the geometry and thus on the specimen type. The tests and test results discussed in this paper are determined with an $\operatorname{ESE}(T)$ specimen. Figure 2 shows this $\operatorname{ESE}(T)$ specimen and the used dimensions. The stress intensity factor range is proportional to the load range, depends on the crack length and the type of geometry. The specific formula of $\Delta K$ for an $\operatorname{ESE}(T)$ specimen (which can be found in [3]) is:

$$
\Delta K=[\Delta P /(B \sqrt{W})] F
$$

With $\Delta P$ the load range, $B$ the specimen thickness $(15 \mathrm{~mm}), W$ the specimen width $(60 \mathrm{~mm})$ and $F$ a factor depending on the crack length, for which the exact formula can be found in [3].

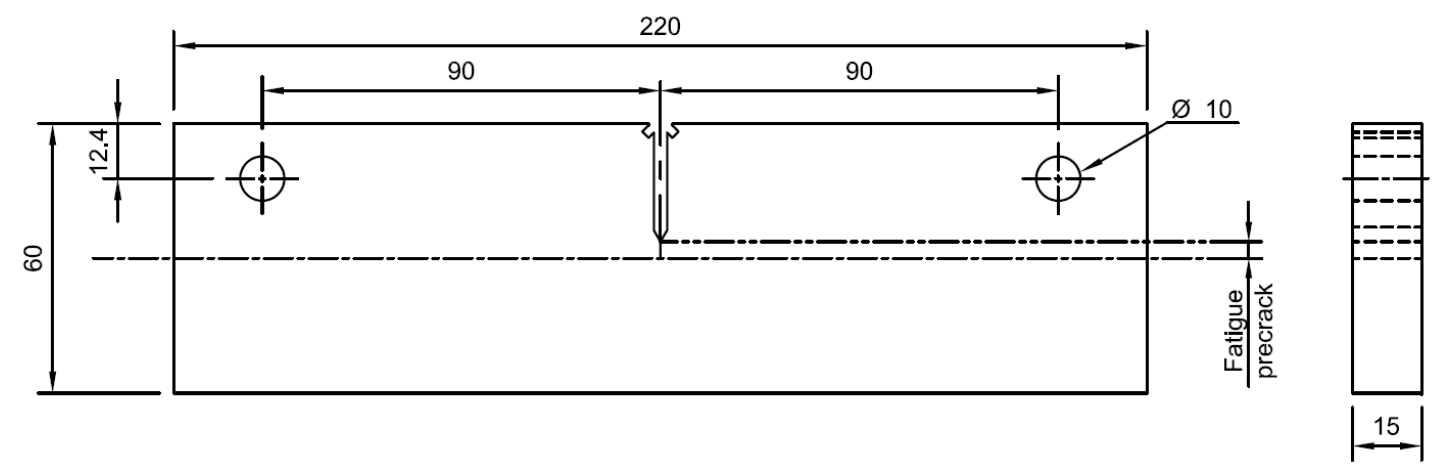

Figure 2: $E S E(T)$ specimen

\subsection{Instrumentation and testing procedure}

For the tests described in the dissertation two instrumentation techniques are used to monitor crack growth. The first one is a clip gauge which is mounted in a machined crack mouth of the specimen (figure 3 (1)). Four strain gauges (two on each leg of the clip gauge) measure strain and thus compliance of the specimen, which is translated in a certain voltage through a Wheatstone bridge. The voltage is converted to a crack mouth opening displacement (CMOD) by calibrating the clip gauge. With the compliance equations (available in [3]) this can be directly linked to a certain crack length. The clip gauge used is a 3541-005M-100M-LT model with $5.00 \mathrm{~mm}$ gauge length which can travel from -1.00 to $10.00 \mathrm{~mm}$.

For an ESE(T) specimen, the crack length can be calculated using the expressions for front-face compliance:

$$
\begin{gathered}
a / W=M_{0}+M_{1} U+M_{2} U^{2}+M_{3} U^{3}+M_{4} U^{4}+M_{5} U^{5} \\
U=\left[\left(\frac{E B v_{0}}{\Delta P}\right)^{\frac{1}{2}}+1\right]^{-1}
\end{gathered}
$$

With $E$ the Young's modulus, $B$ the specimen thickness, $v_{0}$ the $C M O D$ and $\triangle P$ the load range. $M_{0}, M_{1}, M_{2}, M_{3}$, $\mathrm{M}_{4}$ and $\mathrm{M}_{5}$ are constants that can be found in [3].

Direct current potential drop (DCPD) is used as a second measurement technique. A constant current is sent through the specimen and as the resistance of the specimen increases when the uncracked ligament of the specimen becomes smaller, the measured voltage also increases. This voltage can be linked to a certain crack length with the formula (which can be found in [3]):

$$
a=\frac{W}{\pi} \operatorname{Cos}^{-1}\left(\frac{\operatorname{Cosh}\left(\frac{\pi}{W} Y_{0}\right)}{\operatorname{Cosh}\left(\frac{V}{V_{r}} \operatorname{Cosh}^{-1}\left(\frac{\operatorname{Cosh}\left(\frac{\pi}{W} Y_{0}\right)}{\operatorname{Cos}\left(\frac{\pi}{W} a_{r}\right)}\right)\right)}\right)
$$

With a the crack length, $W$ the specimen width, $Y_{0}$ the distance between measurement pins (see next paragraph), $\mathrm{V}$ the measured voltage, $\mathrm{ar}_{\mathrm{r}}$ a reference crack size from another measurement method and $\mathrm{Vr}$ the corresponding voltage for this reference crack length. 
The direct current power source used was an auto ranging Farnell AP60-150 set at 35 Amperes. The measurement instrument used was a nanovolt meter Agilent 34420 with a continuous integrating measurement method (Multi-slope III A-D converter) and a -D Linearity of $0.00008 \%$ of reading $+0.00005 \%$ of range. Figure 4 shows the connections needed for potential drop. At the top and the bottom (2 and 3 ) the current is introduced and connected to earth. 4 and 5 are measurement pins, used to measure the potential difference over the crack mouth. 6 denotes the reference pins, which measure a reference potential difference to filter out environmental effects, such as temperature changes. 7 indicates reference lines that are used to visually confirm the crack length that is reported by the different instrumentation techniques.

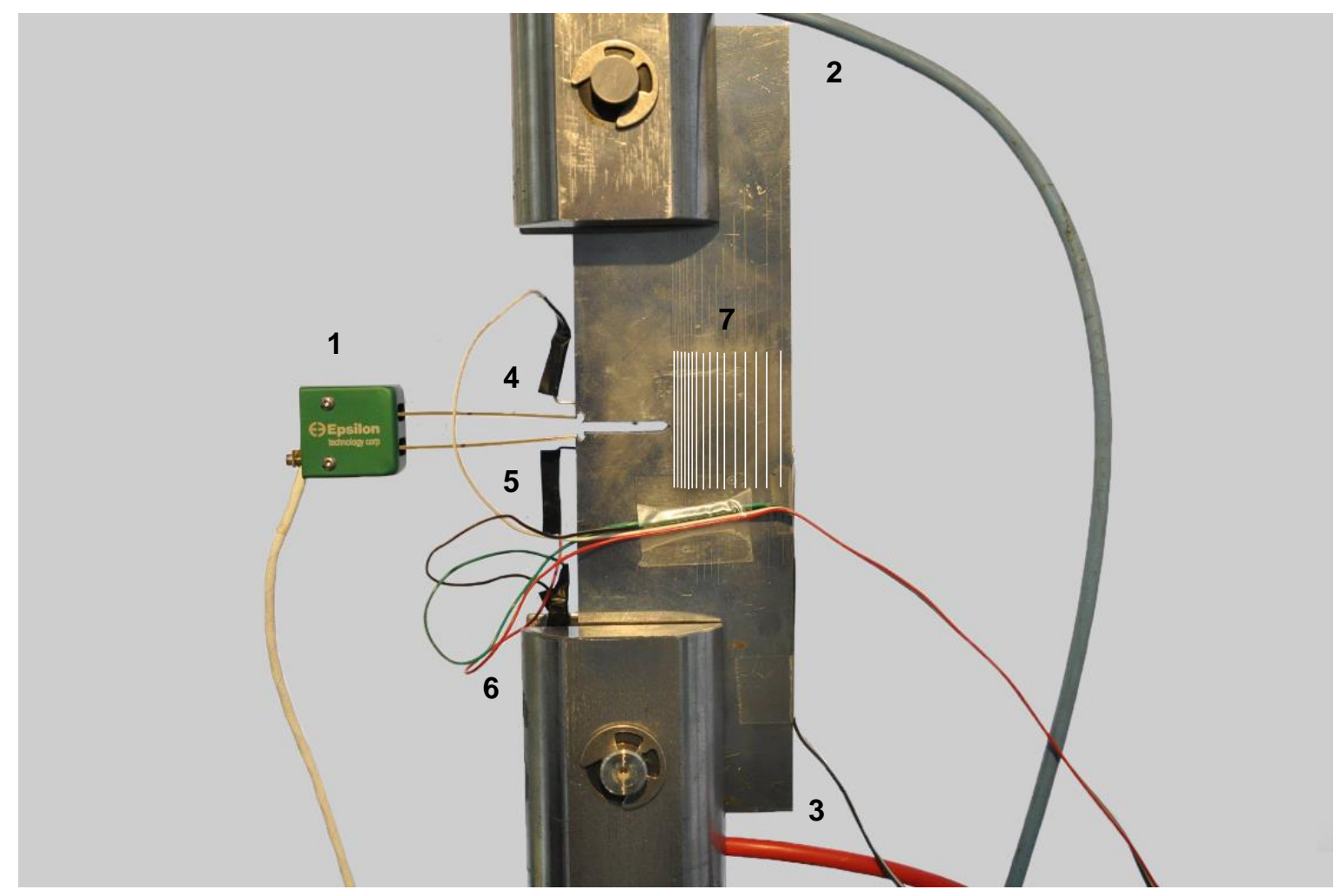

Figure 3: Illustration of instrumented ESE(T) specimen. Crack growth is monitored by clip gauge and potential drop measurements.

\section{4 a/W-N curve}

The comparison of the two instrumentation methods mentioned can be done with either the Paris law curve or by means of an a/W-N curve. An a/W-N curve is shown in figure 4 where both $\mathrm{K}$-decreasing and $\mathrm{K}$ increasing are combined. Besides the two instrumentation techniques a visual confirmation is performed, where the most important points that were detected at the beginning and end of the test are shown (as recommended by standard [3]). Both methods have an excellent correlation, except for the last and initial part of the test where there is a small deviation between them.

At the start of both $\mathrm{K}$-decreasing and $\mathrm{K}$-increasing there is a small deviation between both methods. The clip gauge results were checked visually multiple times (based on the reference lines in figure 3 ) and this had a good correlation with the actual crack length for both tests (K-decreasing and $\mathrm{K}$-increasing). This means that the potential drop underestimated the crack growth at the beginning of both tests. The potential drop calculation is based on an input of the initial voltage for a certain crack growth and therefore initially at the start-up of a test, the correlation can be a bit deviated. For the rest of the K-decreasing the potential drop readings are close to perfect. For the K-increasing, the DCPD also has a small deviation at the end. Besides the initial voltage and crack growth also the initial pin distance (paragraph 3.3) is an input of the potential drop equation. Therefore the correlation at larger crack growth rates might be less accurate as the pin distance becomes larger. But as can be seen from figure 4, these are minor deviations and the two instrumentation techniques give a very good a/W-N curve. 


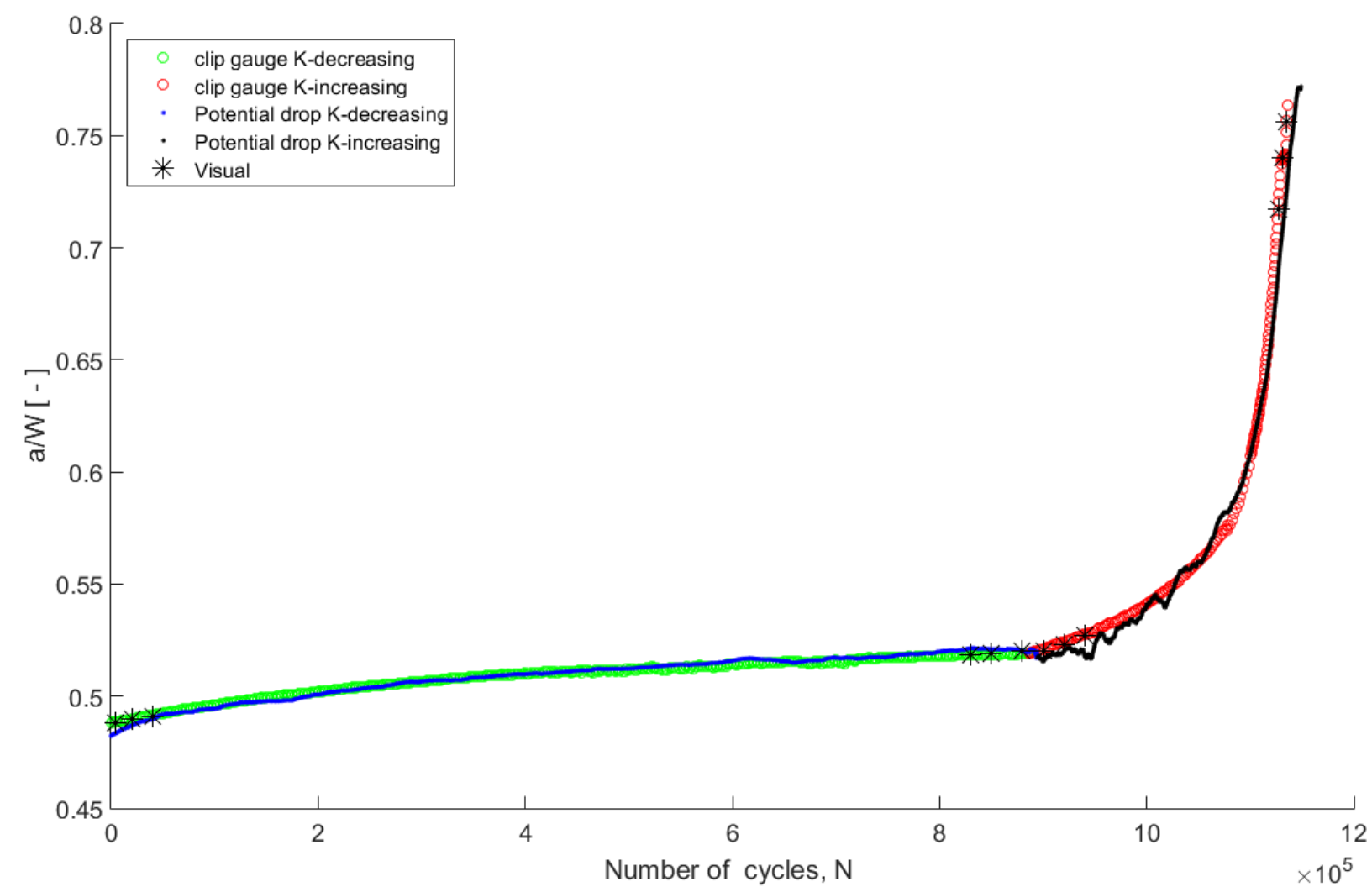

Figure 4: a/W-N curve with both K-decreasing and K-increasing parts determined using potential drop and clip gauge

\subsection{Paris law curve}

As was mentioned above, for both the K-decreasing and K-increasing method, a series of da/dN measurements are taken per $\Delta \mathrm{K}$ block. To obtain the value of da/dN the specified $\Delta \mathrm{a}$ is logically divided by the amount of cycles needed to obtain this crack growth. Multiple da/dN values are saved per block in LabVIEW for redundancy, with a constant crack length for every measurement per block. Every block change this crack length is decreased/increased for respectively the decreasing and increasing method. In case of the $\mathrm{K}$-increasing this is done because crack growth becomes so fast that a larger $\Delta \mathrm{a}$ is needed to assure a stable $\mathrm{da} / \mathrm{dN}$ measurement. For the $\mathrm{K}$-decreasing the $\Delta \mathrm{a}$ is lowered because da/dN values become so small that it is too time-consuming to obtain certain crack growth.

The program makes it possible to obtain a Paris law curve with a single specimen, but of course multiple tests can be done for redundancy. The averaged results for both clip gauge and potential drop are shown in figure 4. In the stable propagation phase similar da/dN values for both DCPD and clip gauge are obtained. The standard requires a value lower than $10^{-7} \mathrm{~mm} /$ cycle crack growth to obtain the threshold stress intensity factor range, which takes a long time to obtain. Even though a few points in the neighbourhood were obtained and a clear trend is observed. This means that the threshold is around 5-6 $M P a * \sqrt{m}$. A typical HSLA steel according to [7] has indeed, for a stress ratio of 0.1 , a $\triangle \mathrm{K}_{\text {threshold }}$ of around 4-6 $M P a * \sqrt{m}$ dependent on the material characteristics. The stable propagation phase is clearly seen with both instrumentation techniques for $\Delta \mathrm{K}$ values ranging from 10 to $50 M P a * \sqrt{m}$.

It is clear that both methods have an excellent correlation. At both the threshold and the higher region of the curve, there is more deviation. The deviation at the end of the $\mathrm{K}$-increasing test was already observed and discussed for the a/W-N curve as well. Based on these observations, the scatter for the higher $\Delta \mathrm{K}$ values can be explained. At the threshold it was observed that, in general, there was more scatter in the crack growth rate. This might explain differences between instrumentation. 


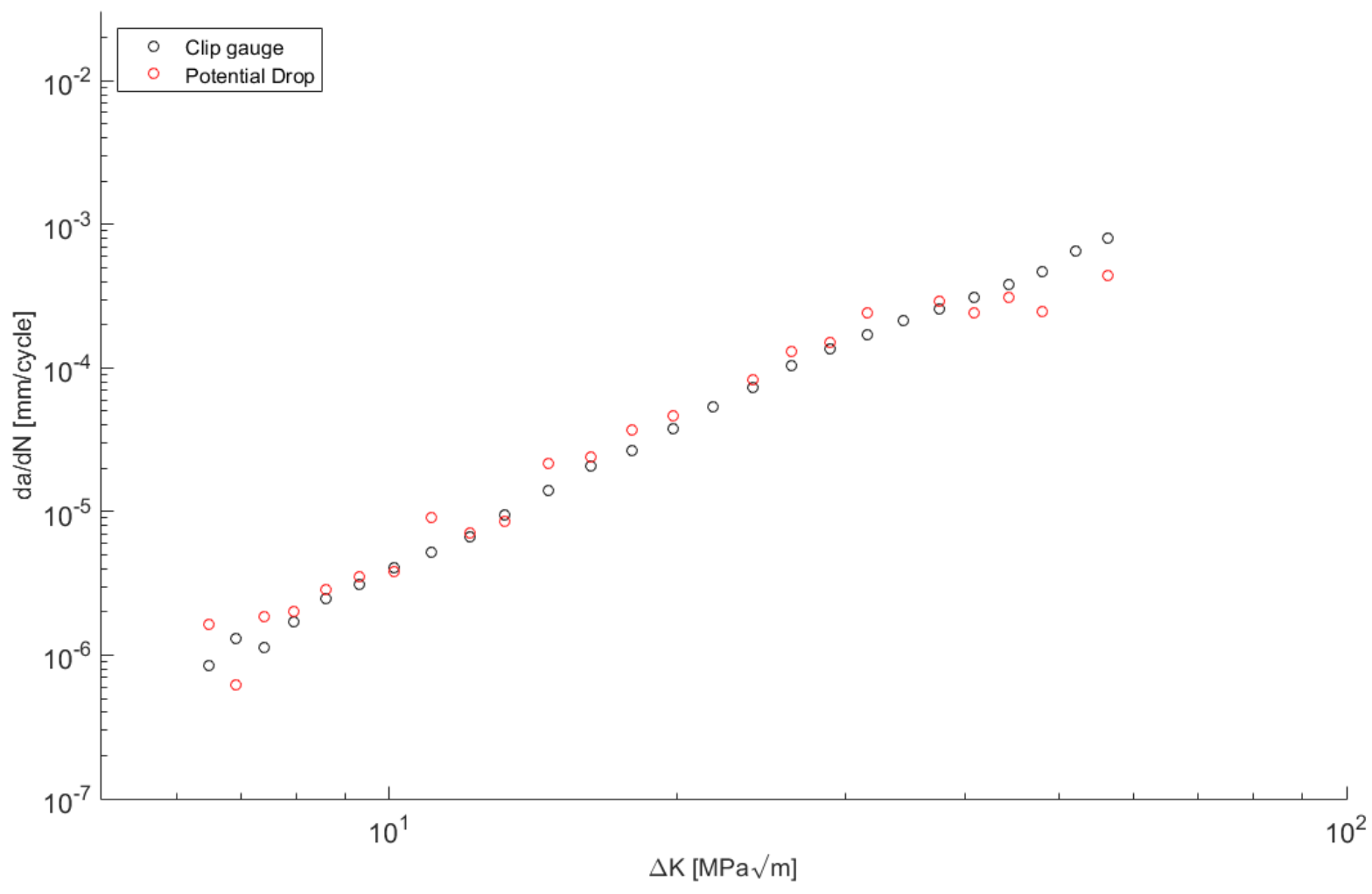

Figure 5: Paris law curve obtained from DCPD (red) and clip gauge (black) readings

\section{CONCLUSIONS}

In this work, a $\triangle \mathrm{K}$ control test was performed in order to obtain the Paris law curve of an HSLA steel making use of both clip gauge and DCPD instrumentation techniques. For this purpose, a self-developed LabVIEW program was used, that allows to control a test from the voltage readings of the clip gauge. It makes it possible to obtain an entire Paris law curve with only one specimen. In parallel, DCPD was used to correlate the measured voltage with the crack growth. A specimen was subjected to both a K-decreasing and K-increasing test. The resulting a/W-N and Paris law curve were plotted and used to compare the two instrumentation techniques. Based on these two curves, they show a good correlation with both each other and a visual confirmation. Even though there was a bit more scatter in the initial potential drop readings, it is a very promising method, which can eventually also be used as an online method to control a test.

\section{REFERENCES}

[1] Micone, N., De Waele, W. (2015). Comparison of Fatigue Design Codes with Focus on Offshore Structures. In International Conference on Ocean, Offshore and Artic Engineering. Canada, May 31 June 5. Ghent University, Soete Laboratory: ASME.

[2] Klysz, S., Leski, A. (2012) 'Good Practice for Fatigue Crack Growth Curves Description', in Belov, A. (ed.) Applied Fracture Mechanics. InTech, pp. 197-200.

[3] Standard test method for measurement of fatigue crack growth rates, ASTM E647. ASTM International, West Conshohocken, USA, 2013.

[4] Černý, I (2004). "The use of DCPD method for measurement of growth of cracks in large components at normal and elevated temperatures". Engineering Fracture Mechanics 71 (2004) 837-848.

[5] Jacobsson L, Persson S., Melin S. (2009)."SEM study of overload effects during fatigue crack growth using an image analyzing technique and potential drop measures". Fatigue Fract Eng Mater Struct 33, 105-115.

[6] Micone, N. (2014) Internal report V4: Material Characterization, Ghent University: Labo Soete.

[7] Khlefa A. Esaklul, William W. Gerberich and James P. Lucas, "Near-Threshold Behavior of HSLA Steels," in HSLA Steels-Technology \& Applications. American Society for Metals, Metals Park OH, 1984, p 571 